\title{
Using a Quartic Spline Function for Certain Birkhoff Interpolation Problem
}

\author{
Kulbhushan Singh \\ The Papua New Guinea \\ University of Technology, Lae \\ Papua New Guinea,
}

\author{
Ambrish Kumar Pandey \\ Integral University,Lucknow,
}

\begin{abstract}
Let $\mathrm{f}$ be a real valued function defined in $[0,1]$, with values known at intermediate points such that the first derivatives of $\mathrm{f}$ at all nodes are also known at intermediate points.In this paper, we construct an interpolatory quartic spline s which interpolates the function $\mathrm{f}$. Unique existence and convergence of this spline are also established. This type of construction is known to have found aesthetic utility in finding areas under or bounded by polynomial curves.
\end{abstract}

\section{Keywords}

Lacunary interpolation, spline, diagonal dominance

\section{INTRODUCTION}

Let $\Delta: \mathbf{0}=x_{\mathbf{0}}<\boldsymbol{x}_{\mathbf{1}}<\cdots<\boldsymbol{x}_{n-1}<\boldsymbol{x}_{n}=\mathbf{1}$ be a partition of unit interval $\mathrm{I}=[0,1]$ with $\boldsymbol{x}_{\boldsymbol{k}+\boldsymbol{1}}-\boldsymbol{x}_{\boldsymbol{k}}=\boldsymbol{h}_{\boldsymbol{k}}, \mathrm{k}=0, \ldots, \mathrm{n}-1$. Denote by $\boldsymbol{S}_{N, 4}^{(2)}$ the class of quartic splines s(x) satisfying the condition that $\boldsymbol{s}(\boldsymbol{x}) \in \boldsymbol{C}^{2}(\boldsymbol{I})$ and is quartic in each subintervals of I. In the past this class of splines is used by various authors with different interpolatory conditions, see [6] and [12]. For more related work one can refer [3] - [13]. In this present paper we study the problem in a different perspective where the first derivative of $\mathrm{f}$ is given at intermediate points between the nodes and the first derivative of $\mathrm{f}$ is known at all the nodes, the theorem states as:

\section{THEOREM 1}

If $\mathrm{f}$ is a real valued function of $\mathrm{x}$ defined in $[0,1]$, we have the real numbers $f_{i}^{\prime}, \mathrm{i}=0,1, \ldots, \mathrm{N}+1 ; f_{i+\lambda}, \mathrm{i}=0,1, \ldots, \mathrm{N} ; \mathrm{f}_{0}$ and $\mathrm{f}_{\mathrm{n}+1}$; There exists a unique spline $S_{N, 4}^{(2)}$ such that

$\left.S_{i}^{\prime}=f_{i}^{\prime}, i=0,1, \ldots, N+1\right)$

$\left.S_{i+\lambda}=f_{i+\lambda}, i=0,1, \ldots, N\right\}$

$S_{0}=f_{0}, \quad S_{N+1}=f_{N+1}$,

where $f_{i+\lambda}$ stands for $f\left(x_{i}+\lambda h\right), 0 \leq \lambda \leq 1 ; \lambda \neq(3 \pm \sqrt{3}) / 6$ and $\mathrm{N}$ is even. To avoid lengthy calculations we take $\lambda=\frac{1}{7}$.

\section{PROOF}

If $\mathrm{P}(\mathrm{x})$ is a quartic polynomial in $[0,1]$ then,

$$
\begin{aligned}
P(\mathrm{x})= & P(0) \mathrm{A}(\mathrm{x})+P(1) \mathrm{B}(\mathrm{x})+P^{\prime}(0) \mathrm{C}(\mathrm{x})+P^{\prime}(1) \mathrm{D}(\mathrm{x}) \\
& +P(\lambda) \mathrm{E}(\mathrm{x}) .
\end{aligned}
$$

Using given conditions we obtained the fundamental polynomials $\mathrm{A}(\mathrm{x}), \mathrm{B}(\mathrm{x}), \mathrm{E}(\mathrm{x}), \mathrm{D}(\mathrm{x})$ and $\mathrm{E}(\mathrm{x})$ as follows:

$$
\begin{aligned}
& A(x)=\frac{105}{13} x^{4}-\frac{184}{13} x^{3}+\frac{66}{13} x^{2}+1 \\
& B(x)=\frac{-105}{13} x^{4}+\frac{184}{13} x^{3}-\frac{66}{13} x^{2}
\end{aligned}
$$

$$
\begin{aligned}
& C(x)=\frac{77}{13} x^{4}-\frac{141}{13} x^{3}+\frac{51}{13} x^{2}+x, \\
& D(x)=\frac{28}{13} x^{4}-\frac{43}{13} x^{3}+\frac{15}{13} x^{2} \\
& E(x)=\frac{49}{26} x^{4}-\frac{49}{13} x^{3}+\frac{49}{26} x^{2}
\end{aligned}
$$

For further calculations we have

$$
\begin{array}{ll}
A^{\prime \prime}(0)=\frac{132}{13} ; & A^{\prime \prime}(1)=\frac{288}{13} ; \\
B^{\prime \prime}(0)=\frac{-132}{13} ; & B^{\prime \prime}(1)=\frac{-288}{13} ; \\
C^{\prime \prime}(0)=\frac{102}{13} ; & C^{\prime \prime}(1)=\frac{180}{13} ; \\
D^{\prime \prime}(0)=\frac{30}{13} ; & D^{\prime \prime}(1)=\frac{108}{13} ; \\
E^{\prime \prime}(0)=\frac{49}{13} ; & E^{\prime \prime}(1)=\frac{49}{13}
\end{array}
$$

Set $\mathrm{x}=\mathrm{x}_{\mathrm{i}}+$ th, $0 \leq \mathrm{t} \leq 1$, then the quartic spline $\mathrm{s}$ which satisfies (2.1) in $\left[\mathrm{x}_{\mathrm{i}}, \mathrm{x}_{\mathrm{i}+1}\right]$ can be expressed as

$\mathrm{s}(\mathrm{x})=\mathrm{s}_{\mathrm{i}} \mathrm{A}(\mathrm{t})+\mathrm{S}_{\mathrm{i}+1} \mathrm{~B}(\mathrm{t})+\mathrm{h} f_{i}^{\prime} \mathrm{C}(\mathrm{t})+\mathrm{h} f_{i+1}^{\prime} \mathrm{D}(\mathrm{t})+\mathrm{h}^{2} f_{i+\lambda}^{\prime} \mathrm{E}(\mathrm{t})$

We have the similar expression for $\mathrm{s}$ in $\left[\mathrm{x}_{\mathrm{i}-1}, \mathrm{x}_{\mathrm{i}}\right]$ since $\mathrm{s} \in \mathrm{C}^{2}[0,1]$ then

$$
s^{\prime \prime}\left(x_{i}+\right)=s^{\prime \prime}\left(x_{i}-\right) ; \quad \mathrm{i}=1,2 \ldots, \mathrm{N} .
$$

Leads the following system of equation $-24 \mathrm{~s}_{\mathrm{i}-1}+35 \mathrm{~s}_{\mathrm{i}}-11 \mathrm{~s}_{\mathrm{i}+1}=\frac{h}{2}\left(30 f_{i-1}^{\prime}+f_{i}^{\prime}+5 h f_{i+1}^{\prime}+\right.$

$$
+\frac{49}{12} h^{2}\left(f_{i-1+\lambda}^{\prime \prime}-f_{i+\lambda}^{\prime \prime}\right)
$$

where,

$$
\begin{gathered}
s^{\prime \prime}\left(x_{i}+\right)=\mathrm{s}_{\mathrm{i}} A^{\prime \prime}(0)+\mathrm{S}_{\mathrm{i}+1} B^{\prime \prime}(0)+\mathrm{h} f_{i}^{\prime} C^{\prime \prime}(0)+ \\
+\mathrm{h} f_{i+1}^{\prime} D^{\prime \prime}(0)+\mathrm{h}^{2} f_{i+\lambda}^{\prime \prime} E^{\prime \prime}(0) \\
s^{\prime \prime}\left(x_{i}-\right)=\mathrm{s}_{\mathrm{i}-1} A^{\prime \prime}(1)+\mathrm{S}_{\mathrm{i}} B^{\prime \prime}(1)+\mathrm{h} f_{i-1}^{\prime} C^{\prime \prime}(1)+ \\
+\mathrm{h} f_{i}^{\prime} D^{\prime \prime}(1)+\mathrm{h}^{2} f_{i-1+\lambda}^{\prime \prime} E^{\prime \prime}(1) .
\end{gathered}
$$

Clearly it is diagonally dominant so existence is unique.

An interesting particular case is for $\lambda=0$ (i.e. $f_{0}, f_{N+1}, f_{i}^{\prime}$ and $\mathrm{f}_{\mathrm{i}}^{\prime \prime}, \mathrm{i}=0,1, \ldots, \mathrm{N}$ are known); (2.3) is then reduced to the recurrence formula 
$-\mathrm{s}_{\mathrm{i}-1}+\mathrm{s}_{\mathrm{i}}=(\mathrm{h} / 2)\left(\mathrm{f}_{\mathrm{i}-1}^{\prime}+\mathrm{f}_{\mathrm{i}}^{\prime}\right)+\left(\mathrm{h}^{2} / 12\right)\left(\mathrm{f}_{\mathrm{i}-1}^{\prime}+\mathrm{f}_{\mathrm{i}}^{\prime}\right)$,

with $s_{0}=f_{0}$. Hence $s_{i}, i=1,2, \ldots, N$ can also be computed through the following formula

$\mathrm{s}_{\mathrm{i}}=\mathrm{f}_{0}+(\mathrm{h} / 2)\left(\mathrm{f}_{0}^{\prime}+\mathrm{f}_{\mathrm{i}}^{\prime}+2 \sum_{j=1}^{i-1} f_{j}^{\prime}\right)+\left(\mathrm{h}^{2} / 12\right)\left(\mathrm{f}_{0}^{\prime \prime}+\mathrm{f}_{\mathrm{i}}^{\prime \prime}\right)$

\section{ERROR ESTIMATES}

In this section an $\mathrm{L}_{\infty}$ error estimate for the above interpolating quartic spline for $\lambda=0$ (i.e. in addition to the boundary conditions $\mathrm{f}_{0}, \mathrm{f}_{\mathrm{N}+1}$, the first and second derivatives are known at the nodes except for $f_{N+1}^{\prime \prime}$ ) is represented. For the other values of $\lambda$ such an estimate is an open problem.

\section{THEOREM 2}

Let $\mathrm{s}$ be the quartic spline as defined above, with $\lambda=0$. If $\mathrm{f} \epsilon$ $C^{l}[0,1], l=2,3,4$, then for any $\mathrm{x} \in[0,1]$

$\left|\mathrm{s}^{(\mathrm{r})}(\mathrm{x})-\mathrm{f}^{(\mathrm{r})}(\mathrm{x})\right| \leq\left\{\begin{array}{cc}C_{l} h^{l-r} w_{l}(h), & r=1,2 \\ \left(\frac{C_{l}}{2}\right) h^{l-1} w_{l}(h), & r=0 .\end{array}\right.$

Furthermore, if $\mathrm{f} \in \mathrm{C}^{5}[0,1]$ then for any $\mathrm{x} \in[0,1]$

$\left|\mathrm{s}^{(\mathrm{r})}(\mathrm{x})-\mathrm{f}^{(\mathrm{r})}(\mathrm{x})\right| \leq \begin{cases}C_{l} h^{5-r}\left\|f^{(5)}\right\| \infty, & r=1,2 \\ \left(\frac{C_{l}}{2}\right) h^{4}\left\|f^{(5)}\right\| \infty, & r=0 .\end{cases}$

Where $\omega_{l}(\mathrm{~h})$ is the modulus of continuity of $\mathrm{f}^{(l)}$; $\omega_{l}(\mathrm{~h}) \leq 2\left\|f^{(l)}\right\|_{\infty}$ and

$\mathrm{C}_{2}=20, \mathrm{C}_{3}=8, \mathrm{C}_{4}=5 / 2, \mathrm{C}_{5}=31 / 30$.

\section{PROOF}

We give the proof of this theorem for $l=3$ and $l=5$ since it runs along the same line for the other cases.

Set $\mathrm{e}_{\mathrm{i}}=\mathrm{s}\left(\mathrm{x}_{\mathrm{i}}\right)-\mathrm{f}\left(\mathrm{x}_{\mathrm{i}}\right)$ then from $(2,4)$ we have

$-\mathrm{e}_{\mathrm{i}-1}+\mathrm{e}_{\mathrm{i}}=(\mathrm{h} / 2)\left(f_{i-1}^{\prime}+f_{i}^{\prime}\right)+\left(\mathrm{h}^{2} / 12\right)\left(f_{i-1}^{\prime \prime}+f_{i}^{\prime \prime}\right)+f_{\mathrm{i}-1}-f_{\mathrm{i} ;} \mathrm{i}=$ $1,2, \ldots, \mathrm{N}$.

Expand the right hand side around $\mathrm{x}_{\mathrm{i}}$ by Taylor's expansion to get

$$
-\mathrm{e}_{\mathrm{i}-1}+\mathrm{e}_{\mathrm{i}}=\left\{\begin{array}{cc}
\left(h^{3} / 4\right)\left[f^{(3)}\left(\alpha_{i}\right)-f^{(3)}\left(\beta_{i}\right)\right], & l=3 \\
\left(h^{5} / 48\right)\left[f^{(5)}\left(\alpha_{i}\right)-\left(h^{5} / 45\right) f^{(5)}\left(\beta_{i}\right)\right], l=5, & =5
\end{array}\right.
$$

where $\alpha_{\mathrm{i}}, \beta_{\mathrm{i}} \epsilon\left(\mathrm{x}_{\mathrm{i}-1}, \mathrm{x}_{\mathrm{i}}\right), \mathrm{e}_{\mathrm{o}}=0$. If follows then for $\mathrm{i}=1,2, \ldots \mathrm{N}$

$\left|-e_{i-1}+e_{i}\right| \leq \begin{cases}\left(h^{3} / 4\right) \omega_{3}(h) & l=3 \\ \left(31 h^{5} / 720\right)\left\|f^{(5)}\right\| \infty, & l=5\end{cases}$

From (2.2) we have

$\mathrm{h}^{2}\left[s^{\prime \prime}(\mathrm{x})-f^{\prime \prime}(\mathrm{x})\right]=\mathrm{e}_{\mathrm{i}} A^{\prime \prime}(t)+e_{i+1} B^{\prime \prime}(t)+\Delta_{i}$

where

$$
\begin{gathered}
\Delta_{i}=h f_{i}^{\prime} C^{\prime \prime}(t)+h f_{i+1}^{\prime} D^{\prime \prime}(t)+h^{2} f_{i}^{\prime \prime} E^{\prime \prime}(t)+f_{i} A^{\prime \prime}(t)+ \\
+f_{i+1} B^{\prime \prime}(t)-h^{2} f^{\prime \prime}(x) .
\end{gathered}
$$

Using Taylor's expansions of order $l$ around $\mathrm{x}_{\mathrm{i}}$ and the following identities

$$
\begin{gathered}
A^{\prime \prime}(x)+B^{\prime \prime}(x) \equiv 0, B^{\prime \prime}(x)+C^{\prime \prime}(x)+D^{\prime \prime}(x) \equiv 0, \\
B^{\prime \prime}(x)+2 D^{\prime \prime}(x)+2 E^{\prime \prime}(x) \equiv 2,
\end{gathered}
$$

$$
\begin{aligned}
& B^{\prime \prime}(x)+3 D^{\prime \prime}(x)+6 \lambda E^{\prime \prime}(x) \equiv 6 x, \\
& B^{\prime \prime}(x)+4 D^{\prime \prime}(x)+12 \lambda^{2} E^{\prime \prime}(x) \equiv 12 x^{2}
\end{aligned}
$$

to get

$$
\begin{aligned}
& \mid \begin{array}{l}
\left|\Delta_{i}\right| \leq \\
\left(h^{3} / 6\right) \omega_{3}(h)\left(\left|B^{\prime \prime}(t)\right|+3\left|D^{\prime \prime}(t)\right|\right), \quad l=3 \\
\left(h^{5} / 120\right)\left\|f^{(5)}\right\| \infty\left(\left|B^{\prime \prime}(t)\right|+5\left|D_{0}^{\prime \prime}(t)\right|+20 t^{3}\right), l=5 .
\end{array}
\end{aligned}
$$

$\operatorname{But} B^{\prime \prime}(t) \leq 12,\left|D^{\prime \prime}(t)\right| \leq 6,\left|E^{\prime \prime}(t)\right| \leq 1$,

$0 \leq t \leq 1$, therefore (3.5), (3.4) and (3.3) give

$\left|s^{\prime}(\mathrm{x})-f^{\prime}(\mathrm{x})\right| \leq \begin{cases}8 h \omega_{3}(h), & l=3 \\ \left(\left(31 h^{3}\right) / 30\left\|f^{(5)}\right\| \infty,\right. & l=5\end{cases}$

Integrating once over $\left[\mathrm{x}_{\mathrm{i}}, \mathrm{x}\right]$, using $s^{\prime}\left(\mathrm{x}_{\mathrm{i}}\right)-f^{\prime}\left(\mathrm{x}_{\mathrm{i}}\right)=0$ and then second times over $[0, x]$ (resp. over $[\mathrm{x}, 1])$ if $\mathrm{x}$ is closer to 0 (resp. to 1 ), using $\mathrm{s}(0)-\mathrm{f}(0)=0$ ( resp. $\mathrm{s}(1)-\mathrm{f}(1)=0$ ), these steps will complete the proof.

Notice that

$\mathrm{F}_{\mathrm{i}}=\mathrm{f}_{0}+\int_{0}^{x_{i}} f^{\prime} d x, i=0,1, \ldots, \mathrm{N}$.

Using (2.4) for $\mathrm{i}=1,2, \ldots, \mathrm{N}$, we have

$s_{i} \quad f_{i}=\left[(h / 2)\left(f_{0}^{\prime}+f_{i}^{\prime}+2 \sum_{j=1}^{i-1} f_{i}\right)+\left(h^{2} / 12\right)\left(f_{0}^{\prime}+f_{i}^{\prime}\right)\right]-$ $\int_{0}^{x_{i}} f^{\prime}(x) d x$.

The right hand side is in fact the error of the corrected trapezoidal rule applied to the function $f^{\prime}(\mathrm{x})$ in $\left[0, \mathrm{x}_{\mathrm{i}}\right]$. Therefore (Carl de Boor [2], p. 321)

$\left|\mathrm{s}_{\mathrm{i}}-\mathrm{f}_{\mathrm{i}}\right| \leq\left(\mathrm{h}^{4} / 720\right)\left\|f^{(5)}\right\| \infty, \quad i=1,2, \ldots, N, \mathrm{f} \in \mathrm{C}^{5}[0,1](3.7)$

Furthermore, notice that $s^{\prime}(x)$ is a cubic Hermite interpolant for $f^{\prime}$ in $\left[\mathrm{x}_{\mathrm{i}}, \mathrm{x}_{\mathrm{i}+1}\right], \mathrm{i}=0,1, \ldots, \mathrm{N}-1$. Since $s^{\prime}(x)$ is a cubic polynomial in $\left[\mathrm{x}_{\mathrm{i}}, \mathrm{x}_{\mathrm{i}+1}\right]$, $s_{i}^{\prime}=f_{i}^{\prime}, \quad s_{i+1}^{\prime}=$ $f_{i+1}^{\prime}, s_{i}^{\prime}=f_{i}^{\prime}$, and $s_{i+1}^{\prime}=f_{i+1}^{\prime}$ (this is not the case in the last sub-interval since $f_{N+1}^{\prime \prime}$ is not available). Therefore, using the classical error of Hermite interpolation, we have (Carl de Boor [2], p. 288)

$\left|\mathrm{s}^{\prime}(\mathrm{x})-\mathrm{f}(\mathrm{x})\right| \leq\left(\mathrm{h}^{4} / 384\right)\left\|f^{(5)}\right\|_{\infty}, \forall \mathrm{x} \in\left[0, \mathrm{x}_{\mathrm{N}}\right], \mathrm{f} \in \mathrm{C}^{5}[0,1]$

Integrating over $[0, \mathrm{x}]$, using $\mathrm{s}(0)-\mathrm{f}(0)=0$, we obtain,

$|\mathrm{s}(\mathrm{x})-\mathrm{f}(\mathrm{x})| \leq\left(\mathrm{h}^{4} / 384\right)\left\|f^{(5)}\right\|_{\infty}, \forall \mathrm{x} \in\left[0, \mathrm{x}_{\mathrm{N}}\right]$

The upper bound in (3.8)(resp. (3.9)] is about 400 (resp. 200) times less than the corresponding bound given in (3.2).

In the last interval $\left[\mathrm{x}_{\mathrm{N}}, \mathrm{x}_{\mathrm{N}+1}\right]$, integrating (3.6) once over $\left[\mathrm{x}_{\mathrm{N}}\right.$, $\left.\mathrm{x}_{\mathrm{N}+1}\right]$, using $s^{\prime}\left(\mathrm{x}_{\mathrm{N}}\right)-f^{\prime}\left(\mathrm{x}_{\mathrm{N}}\right)=0$ and then second times over $[\mathrm{x}, 1]$ using $\mathrm{s}(1)-\mathrm{f}(1)=0$, we get

$$
\left|\mathrm{s}^{\prime}(\mathrm{x})-\mathrm{f}(\mathrm{x})\right| \leq\left(31 \mathrm{~h}^{4} / 30\right)\left\|f^{(5)}\right\|_{\infty}, \mathrm{x} \in\left[\mathrm{x}_{\mathrm{N}}, 1\right]
$$




$$
|\mathrm{s}(\mathrm{x})-\mathrm{f}(\mathrm{x})| \leq\left(31 \mathrm{~h}^{4} / 30\right)\left\|f^{(5)}\right\|_{\infty}, \mathrm{x} \in\left[\mathrm{x}_{\mathrm{N}}, 1\right]
$$

This proves the theorem. The case $\lambda=1$ is very similar to that of $\lambda=0$.

\section{CONCLUSION}

We have studied the existence and uniqueness of a quartic spline that matches with the first derivates of $f(x)$ at the nodes and the second derivatives between them. When both the first and second derivatives are given at the nodes and error estimates derived, which, together with the numerical results, showed the method to be efficient.

\section{REFERENCES}

[1] Ahlberg, J. H., Nilson, E. N., and Walsh, J. L. The theory of Splines and their Applications, Academic Press, New York, 1967.

[2] Boor, Carl de, A Practical Guide to splines, SpringerVerlag 1978.

[3] Mathur, K. K., and Saxena, A. Odd degree splines of higher order, Acta Math. Hung., 62 (3 - 4) (1993), 263 275 .

[4] Burkett, J. and Verma, A. K. On Birkhoff Interpolation $(0 ; 2)$ case, Aprox. Theory and its Appl. 11(2) (1995), $59-66$.

[5] Saxena, A., and Singh, Kulbhushan Lacunary
Interpolation by Quintic splines, Journal of Indian Mathematical Society, Vol.66 No.1-4 (1999), 23-33.

[6] Singh, Kulbhushan Interpolation by quartic splines, African Jour. of Math. and Comp. Sci. Vol. 4 (10), pp. $329-333, \quad 15 \quad$ September, 2011 http://www.academicjournals.org/AJMCSR ISSN 20069731 .

[7] Prasad, J., and Verma, A. K. Lacunary interpolation by quintic splines SIAMJ. Numer.Anal.16, (1979) 10751079.

[8] Singh, Kulbhushan Lacunary odd degree splines of higher order, Proceedings of Conference:Mathematical Science and Applications, Dec. 26-30, 2012, AbuDhabi, UAE.

[9] Saxena, A. Birkhoff interpolation by quintic spline, Annales Univ. Sci. Budapest, 33, (1990) 000-000.

[10] Saxena, R.B. Lacunary Interpolation by quintic spline, SIAM J. Numer. Anal.16, No.6, (1963) 1075- 1079.

[11] Saxena, R. B. On mixed type Lacunary Interpolation II, Acta. Math. Acad. Sci. Hung. 14, (1963)1-19.

[12] Saxena, R. B. and T. C. Joshi, On quartic spline Interpolation, Ganita 33, No. 2 , (1982) 97-111.

[13] Sallam, S. On interpolation by quintic Spline, Bull. Fac. Sci. Assiiut. Univ, 11(1), (1982) 97- 106. 